\title{
Dynamic Regulation of the Kv2.1 Voltage-Gated Potassium Channel during Brain Ischemia through Neuroglial Interaction
}

\author{
Hiroaki Misonou, ${ }^{1}$ Scott M. Thompson, ${ }^{2}$ and Xiang Cai ${ }^{2}$ \\ ${ }^{1}$ Department of Biomedical Science, Dental School, and 2Department of Physiology, School of Medicine, University of Maryland, Baltimore, Maryland \\ 21201
}

\begin{abstract}
The physiological significance of neuroglial interactions in the CNS has been emphasized in neurological conditions such as epilepsy and brain ischemia. The Kv2.1 voltage-gated potassium channel is unique in its ability to form large clusters in the plasma membrane of neuronal cell bodies. We have previously shown that brain ischemia causes rapid dephosphorylation of Kv2.1 subunits and resultant activation of the ion channel function. However, the physiological significance of the channel clustering is unknown. Here we present evidence that clustered Kv2.1 channels in the neuronal plasma membrane are juxtaposed to axosomatic synapses and associated with astrocytic processes expressing high levels of glutamate transporters. In acute cortical slices, ischemic stress rapidly resulted in the dephosphorylation and dispersion of Kv2.1. Selective inhibition of metabolism in astrocytes was sufficient to induce Kv2.1 dephosphorylation in neurons. Interestingly, these effects were blocked by the antagonists of ionotropic glutamate receptors, indicating the involvement of glutamate as the signal mediator between astrocytes and neurons. Furthermore, the pharmacological inhibition of glial glutamate transporter GLT-1 induced the similar Kv2.1 dephosphorylation, whereas exogenous glutamate alone was not efficacious. These results suggest that ischemic stress rapidly causes the dysfunction of glutamate transporters in astrocytes and resultant accumulation of glutamate in the extracellular space. The elevated glutamate may subsequently activate ionotropic glutamate receptors and result in the dephosphorylation of Kv2.1 in neurons. These findings implicate that Kv2.1 clusters are strategically situated at neuroglial junctions to achieve the rapid modulation after ischemic stress via glutamate signaling.
\end{abstract}

Key words: hypoxia; astrocyte; cerebral cortex; phosphorylation; glutamate transporter; neuroprotection

\section{Introduction}

Ischemic stroke is the third most common cause of death (after heart disease and cancers) and a major cause of long-term neurological damage and disability in the United States. Brain ischemia rapidly elicits excess accumulation of the excitatory neurotransmitter, glutamate, which could result from its release from nerve terminals and astrocytes, and the failure in the clearance of glutamate through astrocytic glutamate transporters (Danbolt, 2001). This may result in hyperexcitability of neurons and resultant $\mathrm{Ca}^{2+}$ overload. It has emerged that neurons have developed a diverse array of protective mechanisms against hyperexcitability evoked in brain ischemia using different types of $\mathrm{K}^{+}$channels, such as ATP-sensitive (Yamada et al., 2001; Ballanyi, 2004) and $\mathrm{Ca}^{2+}$-activated $\mathrm{K}^{+}$channels (Wei et al., 2003). In addition to

Received April 3, 2008; revised June 19, 2008; accepted July 23, 2008.

This work was supported by the American Heart Association (H.M.), National Alliance for Research on Schizophrenia and Depression (X.C.), National Institute of Neurological Disorders and Stroke Grant NS40338, and Nationa Institute of Mental Health Grant MH65488 (S.M.T.). We thank Ashley Deitchler and Kaori Misono for technical assistance, Drs. Ke Ren and Kun Yang for the help with slice preparation and initial patch-clamp experiments, and Dr. Patricio $0^{\prime}$ Donnell for the initial slice experiments. We also thank Drs. Yoshitaka Kihira, Ronald Dubner, and Matthew Rasband for critical reading of this manuscript and Dr. Dwight Bergles for helpful discussion.

Correspondence should be addressed to Hiroaki Misonou at the above address. E-mail: hmisono@umaryland.edu.

D01:10.1523/JNEUROSCI.1417-08.2008

Copyright $\odot 2008$ Society for Neuroscience $\quad$ 0270-6474/08/288529-10\$15.00/0 these channels, we have shown that the Kv2.1 voltage-gated $\mathrm{K}^{+}$ channel is likely to be involved in the innate protective response of neurons to the pathological hyperexcitability (Misonou et al., 2004, 2005).

Kv2.1 is a major component of delayed rectifier $\mathrm{K}^{+}$channels in cortical and hippocampal pyramidal neurons (Murakoshi and Trimmer, 1999; Yuan et al., 2002; Pal et al., 2003; Guan et al., 2007 ) and is expressed in the majority of neurons in these brain areas (Trimmer, 1991). Although Kv2.1 shows an extensive cellular expression pattern, its subcellular localization in a neuron is restricted to large clusters on the soma and proximal portion of dendrites (Scannevin et al., 1996; Du et al., 1998). We have recently shown that hyperexcitability caused by ischemic stress results in bulk dephosphorylation of Kv2.1 and the dispersion of the surface channel clusters without substantial internalization of the channels (Misonou et al., 2004, 2005). By using dissociated cultured neurons, we identified that both glutamate and chemically induced ischemic stress (energy depletion) induce Kv2.1 dephosphorylation and require an increase in the intracellular $\mathrm{Ca}^{2+}$ level and resultant activation of calcineurin (Misonou et al., 2004, 2005). Dephosphorylation rapidly leads to changes in the voltage dependency for channel activation toward more hyperpolarized membrane potentials in cultured neurons, which then leads to the dampening of neuronal excitability (Misonou et al., 
2005), i.e., a protective response. However, the physiological significance of Kv2.1 clustering in this mechanism has been largely unexplored.

A previous immunoelectron microscopic study showed that $\mathrm{Kv} 2.1$ is concentrated in the plasma membrane immediately facing astrocytic processes in the somata of pyramidal neurons ( $\mathrm{Du}$ et al., 1998). In the present study, we also provide evidence suggesting the possible morphological association of Kv2.1 clusters and astrocytic processes. Furthermore, our results suggest that Kv2.1 clusters are strategically situated at the junction formed between neuronal and astrocytic plasma membranes to achieve the rapid neuronal protective response after ischemic stress. Brain ischemia results in the dysfunction of astrocytic glutamate transporters and extracellular glutamate accumulation. These changes would lead to the functional modulation of Kv2.1 through specific glutamate receptor signaling, which ultimately results in the feedback suppression of neuronal excitability.

\section{Materials and Methods}

In vivo and in vitro ischemia models. All animal use procedures were performed in strict accordance with the Guide for the Care and Use of Laboratory Animals by the National Institutes of Health. Adult male Sprague Dawley rats (150-300 g; Charles River) were subjected to $\mathrm{CO}_{2}$ inhalation for $2 \mathrm{~min}$ in a closed chamber connected to a $\mathrm{CO}_{2}$ gas cylinder to induce ischemic stress in vivo (Misonou et al., 2005). Control animals were anesthetized by intraperitoneal injection of pentobarbital $(50 \mathrm{mg} /$ $\mathrm{kg}$ ). Animals were then either decapitated to remove the brains or perfused with $4 \%$ paraformaldehyde for immunohistochemistry (see below). We have previously shown that $\mathrm{CO}_{2}$ inhalation and global decapitation ischemia exhibit similar Kv2.1 modulation in vivo.

In vitro ischemic model was established with acute cortical slices. Young male Sprague Dawley rats [postnatal day 24 (P24)-P30] were used. Animals were rapidly decapitated to remove brains. Whole brains were immersed in ice-cold artificial CSF-A (ACSF-A; $125 \mathrm{~mm} \mathrm{NaCl}, 25$ $\mathrm{mm} \mathrm{NaHCO}_{3}, 10 \mathrm{~mm}$ glucose, $3.5 \mathrm{~mm} \mathrm{KCl}, 1.25 \mathrm{~mm} \mathrm{NaH}_{2} \mathrm{PO}_{4}, 0.5 \mathrm{~mm}$ $\mathrm{CaCl}_{2}$, and $3 \mathrm{~mm} \mathrm{MgCl}_{2}, \mathrm{pH} 7.4$ ) and then glued to the stage of a microtome. Slices were cut to a $300 \mu \mathrm{m}$ thickness with a vibrating microtome (Vibratome) in ice-cold ACSF-A bubbled with $95 \% \mathrm{O}_{2}$ and $5 \% \mathrm{CO}_{2}$. They were transferred to ACSF-B, in which $\mathrm{CaCl}_{2}$ is increased to $2 \mathrm{~mm}$ and $\mathrm{MgCl}_{2}$ is decreased to $1 \mathrm{~mm}$. Slices were treated at room temperature with $5 \mathrm{~mm}$ sodium azide and $5 \mathrm{~mm}$ 2-deoxy-D-glucose for chemically induced energy depletion (CED). After the drug treatment, slices were either homogenized in homogenizing buffer $(320 \mathrm{~mm}$ sucrose, $5 \mathrm{~mm}$ phosphate buffer, $\mathrm{pH}$ 7.4) containing a protease inhibitor mixture on ice or fixed with $4 \%$ paraformaldehyde in $0.1 \mathrm{M}$ phosphate buffer ( $\mathrm{PB} ; \mathrm{pH}$ 7.2) for $1 \mathrm{~h}$ at $4^{\circ} \mathrm{C}$

Immunohistochemistry. Rats were anesthetized by the injection of 50 $\mathrm{mg} / \mathrm{kg}$ sodium pentobarbital (i.p.). Animals were then fixed by transcardiac perfusion of $4 \%$ paraformaldehyde in $\mathrm{PB}$. The fixed brain was equilibrated in $30 \%$ sucrose solution for cryoprotection. The brain was sectioned into $40-\mu \mathrm{m}$-thick sagittal brain sections using a cryotome. Cortical slices $(300 \mu \mathrm{m})$ were also sectioned into $40 \mu \mathrm{m}$ sections for immunostaining. Tissue sections were washed in $\mathrm{PB}$ and blocked and permeabilized for $1 \mathrm{~h}$ in $10 \%$ normal goat serum in $\mathrm{PB}$ containing $0.3 \%$ Triton X-100. Sections were then incubated with the following primary antibodies, in combination, overnight at $4^{\circ} \mathrm{C}$ : $\mathrm{K} 89$ monoclonal antibody against Kv2.1 (NeuroMab) (Misonou et al., 2006), anti-Kv2.1 rabbit polyclonal antibody (Trimmer, 1991), anti-GLT-1 mouse monoclonal antibody (BD Biosciences), anti-GLT-1 rabbit polyclonal antibody (Frontier Science) (Tanaka et al., 1997), anti-glial fibrillary acidic protein (GFAP) mouse monoclonal antibody (Millipore Bioscience Research Reagents), N28 mouse monoclonal antibody against vesicular glutamate transporter 1 (VGluT1) (NeuroMab), GAD6 monoclonal antibody for glutamic acid decarboxylase 65 (GAD65) [Developmental Studies Hybridoma Bank (DSHB)] (Kash et al., 1997), anti-glutamate aspartate transporter (GLAST) guinea pig polyclonal antibody (Millipore Bioscience Research Reagents), anti-vesicular acetylcholine transporter
(VAChT) goat polyclonal antibody (Millipore Bioscience Research Reagents) (Muennich and Fyffe, 2004), anti-GlyT2 guinea pig polyclonal antibody (Millipore Bioscience Research Reagents), anti-VMAT2 rabbit polyclonal antibody (Millipore Bioscience Research Reagents), and antiSV2 mouse monoclonal antibody (DSHB) (Sullivan et al., 2004). We have obtained the identical localization pattern with the mouse antibody to that with the rabbit GLT-1 antibody (data not shown), which has been validated with the GLT-1-deficient mice (Tanaka et al., 1997). We mainly used the mouse anti-GLT-1 antibody and not the rabbit antibody because of the availability and the homogeneity of monoclonal antibodies (Rhodes and Trimmer, 2006). Sections were washed and probed with Alexa-dye-conjugated secondary reagents available for each species and IgG isotypes (Invitrogen). After washing, sections were mounted on glass slides and coverslipped for fluorescence imaging. Fluorescent images were taken with a CCD camera installed on a Zeiss Axiovert 200M microscope with $10 \times, 20 \times$, and $63 \times$ lenses and a structured-illumination imaging system, Apotome, using Axiovision software (Zeiss). For semiquantitative immunofluorescence analyses, fluorescence images were obtained with the same exposure time and processed in the same manner throughout the analyses.

Western blot. SDS sample buffer (2\% SDS, 5\% 2-mercaptoethanol, $10 \%$ glycerol, and $62.5 \mathrm{~mm}$ Tris- $\mathrm{HCl}, \mathrm{pH} 6.8$ ) was added to a sample. Proteins were separated in $7.5 \%$ SDS polyacrylamide gels and electrophoretically transferred to nitrocellulose membranes. The membranes were washed, blocked with $4 \%$ nonfat dry milk in $150 \mathrm{~mm} \mathrm{NaCl}, 10 \mathrm{~mm}$ sodium azide, and $20 \mathrm{~mm}$ Tris- $\mathrm{HCl}, \mathrm{pH} 8.0$, for $30 \mathrm{~min}$, and then incubated with primary antibodies overnight in the blocking buffer at $4^{\circ} \mathrm{C}$. We used the K89 mouse monoclonal antibody to detect Kv2.1. This antibody recognizes both phosphorylated and dephosphorylated forms of rat Kv2.1 (Misonou et al., 2006). The membranes were washed and probed with HRP-conjugated secondary antibodies (KPL) according to the manufacturer's instruction. Immunoreactive bands were detected with enhanced chemiluminescence (Perkin-Elmer) and visualized by exposing the membranes to $\mathrm{x}$-ray films. The profiles of optical densities across the multiple Kv2.1-immunoreactive bands resulting from the multiple Kv2.1 species that differ in phosphorylation (Misonou et al., $2005,2006)$ were obtained using ImageJ. We first determined the most phosphorylated fraction of Kv2.1 as a peak around $125 \mathrm{kDa}$ in the profile in the control sample and separated it from other peaks with vertical lines. We then applied the same vertical lines to the profiles of other samples and measured the areas under the curves as the levels of phosphorylated Kv2.1. The ratio of phosphorylated Kv2.1 was given by dividing the level of phosphorylated Kv2.1 by the level of total Kv2.1, which was the area of the total peaks between 90 and $150 \mathrm{kDa}$.

Electrophysiology. $I_{\mathrm{K}}$ current recordings were made with the whole-cell voltage-clamp configuration in acute cortical slices (Cai et al., 2004). Patch pipettes were pulled from borosilicate glass tubing to give a resistance of 4-5 $\mathrm{M} \Omega$ when filled with the pipette solution $(135 \mathrm{~mm}$ K-methanesulfate, $10 \mathrm{~mm} \mathrm{NaCl}, 1 \mathrm{~mm} \mathrm{MgCl}_{2}, 0.1 \mathrm{~mm}$ BAPTA, $2 \mathrm{~mm}$ $\mathrm{Mg}^{2+}$-ATP, $10 \mathrm{~mm}$ phosphocreatine, and $10 \mathrm{~mm}$ HEPES, pH 7.3). Alexa 568 (10 $\mu \mathrm{M}$; Invitrogen) was included in the pipette solution for visualization of the patched cells under fluorescence microscopy. Currents were recorded with a patch-clamp amplifier (Axopatch 200B; Molecular Devices), sampled at $10 \mathrm{kHz}$, and filtered at $2 \mathrm{kHz}$ by a digital Bessel filter. Tetrodotoxin was added to ACSF to block sodium channels. CED reagents were applied to acute cortical slices for $20 \mathrm{~min}$ as a bath application. We chose neurons with apparent pyramidal morphology (one main thick, long apical dendrite with multiple thin proximal dendrites near the soma) under fluorescence microscopy after establishing the whole-cell configuration. Currents were recorded from pyramidal neurons before and after induction of CED. All recordings were performed at room temperature $\left(23-25^{\circ} \mathrm{C}\right)$. The membrane potential was held at $-70 \mathrm{mV}$ and depolarized (or hyperpolarized) from the holding potential of -70 $\mathrm{mV}$ to voltages between -80 and $+60 \mathrm{mV}$ in $10 \mathrm{mV}$ increments for 200 $\mathrm{ms}$. Before the start of the test depolarization, a single prepulse to -10 $\mathrm{mV}$ was given for $30 \mathrm{~ms}$ to inactivate majority of the transient outward $\mathrm{K}^{+}$currents. Linear leak components were identified from the hyperpolarizing pulse and subtracted. Peak outward sustained $\mathrm{K}^{+}$currents $(I)$ were measured $100 \mathrm{~ms}$ into the pulse at each depolarizing potential and 
were converted to conductance $(G)$ using the equation $G=I /\left(V-E_{\mathrm{K}}\right)$. The Nernst $\mathrm{K}^{+}$equilibrium potential $E_{\mathrm{K}}$ was calculated as $-94 \mathrm{mV}$. The normalized conductances were plotted against the test potential $(V)$ and fitted to a single Boltzmann equation $G=G_{\max } /\left(1+\exp \left[-\left(V-G_{1 / 2}\right) /\right.\right.$ $k]$ ), where $G_{\max }$ is the maximum conductance, $G_{1 / 2}$ is the test potential at which the $I_{\mathrm{K}}$ channels have a half-maximal conductance, and $k$ represents the slope of the activation curve.

Data analysis. Data are presented as mean \pm SEM. Statistical analyses were made using Student's $t$ test or ANOVA with Fisher's PLSD post hoc analysis. The sample size of different experiments was estimated by a power analysis using $\mathrm{G}^{\star}$ Power3 (http://www.psycho.uni-duesseldorf.de/ abteilungen/aap/gpower3/) based on our preliminary studies or previous related studies. The power of the sample is at least at a level of $80 \%$. $p<$ 0.05 is considered significant for all cases.

Other materials. Sodium azide, (+)-5-methyl-10,11-dihydro-5Hdibenzo $[a, d]$ cyclohepten-5,10-imine maleate (MK-801), CNQX, fluorocitrate, dihydrokainate (DHK), and NMDA were purchased from Sigma. Threo- $\beta$-benzyloxyaspartate (TBOA) and D,L-AP5 were purchased from Tocris Bioscience. 2-Deoxy-D-glucose was obtained from EMD Biosciences.

\section{Results}

\section{Extrasynaptic localization of Kv2.1 in rat cortical} pyramidal neurons

Kv2.1 forms large ( $\sim 2-\mu \mathrm{m}$-diameter) clusters in the somatodendritic membrane of pyramidal neurons, particularly apparent in the rat primary somatosensory cortex (Trimmer, 1991). Based on the results of Muennich and Fyffe (2004) that Kv2.1 clusters colocalize with the muscarinic cholinergic synapses in spinal motor neurons, we hypothesized that Kv2.1 clusters are also associated with specific synapses in the cerebral cortex. To test the hypothesis, we have immunostained tissue sections of rat cerebral cortex to examine the colocalization of Kv2.1 clusters and several classes of chemical synapses, including cholinergic, glutamatergic, and GABAergic synapses. We decided to look at proteins expressed in presynaptic terminals, including vesicular neurotransmitter transporters because these marker proteins provided us with the specific location at which synapses are formed. By using VAChT as a marker (Muennich and Fyffe, 2004), we confirmed the partial colocalization of Kv2.1 clusters and the cholinergic synapses in motor neurons in spinal cord (data not shown), as reported, and also those in the facial nucleus (Fig. 1A). However, we did not find any overlap of the cholinergic synapses with Kv2.1 clusters in neurons in the somatosensory cortex, because cholinergic axons mostly appeared as varicosities instead of synaptic terminals in the cortex (data not shown).

Instead, our results show that Kv2.1 clusters are localized adjacent to, but not overlapped with, glutamatergic and GABAergic synaptic terminals positive for VGluT1 and GAD65, respectively. As shown in Figure $1 B$, there were many small VGluT1-positive synaptic terminals and large GAD65-positive terminals on the somata of cortical pyramidal neurons (see also supplemental Fig. 3, available at www.jneurosci.org as supplemental material). Kv2.1 clusters were precisely interdigitated with these axosomatic synapses (Fig. $1 B$ ). We also tested other presynaptic markers, VGlyT2 (glycinergic), VMAT2 (monoaminergic), and SV2 synaptic vesicle protein (total synaptic population). None of them overlapped with Kv2.1 clusters (data not shown), even though these markers showed their characteristic expression patterns such as varicosities. These results indicate that Kv2.1 clusters are localized exclusively in the extrasynaptic area of somatic plasma membrane in rat cortical pyramidal neurons.

Importantly, brain ischemia resulted in the dramatic surface dispersion of Kv2.1 clusters (Misonou et al., 2005) and thus released the channels from the extrasynaptic area. In the somatosensory cortex of ischemic brains, Kv2.1 was com- pletely dispersed over the somatic plasma membrane of pyramidal neurons (Fig. 2). The overall staining pattern of GAD65 did not differ in either control or ischemic brains. Quantitative analysis showed that Kv2.1 staining overlapping with GAD65-positive terminals increased from $4.3 \pm 0.6 \%$ (of total) in control to $17.4 \pm 1.3 \%$ in ischemic brains $(p<0.001$; $n=10$ ), indicating that Kv2.1 translocates from extrasynaptic to synaptic membranes during brain ischemia.

\section{Kv2.1 clusters are adjacent to astrocytic processes that express high levels of GLT-1 glutamate transporter}

Together with the previously reported immunoEM study of Kv2.1 (Du et al., 1998), this exclusive extrasynaptic localization of Kv2.1 clusters led us to hypothesize that Kv2.1 clusters are associated with astrocytic processes that come in contact with neuronal membranes in the extrasynaptic zone (Huang and Bergles, 2004). We chose GLT-1 (EAAT2) glutamate transporter as a marker of the astrocytic process, because it is abundant in the cerebral cortex (Regan et al., 2007) and highly expressed in the astrocytic processes (Sullivan et al., 2004). GLT-1 immunostaining showed that in fact GLT-1 is highly expressed in the somatosensory cortex (supplemental Fig. 1, available at www.jneurosci. org as supplemental material) in astrocytes together with GLAST (EAAT1) (supplemental Fig. 2B, available at www.jneurosci. org as supplemental material), another glial glutamate transporter expressed in the cerebral cortex to a lesser extent (Lehre et al., 1995; Regan et al., 2007). High-resolution imaging revealed that GLT-1-positive astrocytic processes wrap around the neuronal cell body to form net-like structures (Fig. 3) (see also Fig. 4) as previously reported. These net-like structures were adjacent to VGluT1-positive and GAD65-positive synaptic terminals and therefore exclusively in the extrasynaptic areas (supplemental Fig. 3, available at www.jneurosci.org as supplemental material) as previously shown with other synaptic markers (Sullivan et al., 2004). Although Kv2.1 and GLT-1 did not substantially colocalize, double-label immunostaining revealed that many of the Kv2.1 clusters ( $65 \pm 10 \%$ in 10 somatosensory layer V pyramidal neurons) are morphologically associated with the GLT-1positive net-like structures (Fig. $3 A, B$ ) in the extrasynaptic area. These results indicate that Kv2.1 clusters are in close proximity to the membranes of astrocytes that express high levels of GLT-1. Again, brain ischemia resulted in the dispersion of Kv2.1, such that Kv2.1 seeps out of the GLT-1-positive extrasynaptic areas and invades synaptic areas (Fig. 3C) (see also Fig. 2).

\section{Ischemic stress in astrocytes results in dephosphorylation of Kv2.1 in neurons}

There is growing evidence that astrocytes contribute to the pathogenesis of brain ischemia at multiple levels (Takuma et al., 2004). Astrocytes may secrete chemical mediators that help neurons survive in damaged tissue, including neurotrophic factors (Tokumine et al., 2003). Because of the potential structural association of Kv2.1 clusters with the GLT-1-positive astrocytic processes (Fig. 3), we hypothesized that brain ischemia compromise the function of astrocytes, which ultimately leads to the modulation of Kv2.1. To test the hypothesis, first we examined the morphological changes in astrocytes in brain ischemia using GFAP as a marker. In control cerebral cortex, there were few astrocytes that expressed detectable levels of GFAP. However, acute ischemic stress in vivo $(\sim 3 \mathrm{~min})$ induced a significant increase of GFAP immunostaining ( $242 \pm 36 \%$ of control; $p=0.02 ; n=3$ ) in the somatosensory cortex (Fig. 4). This increase seemed to be attributable to an increase in the number of astrocytes possessing 


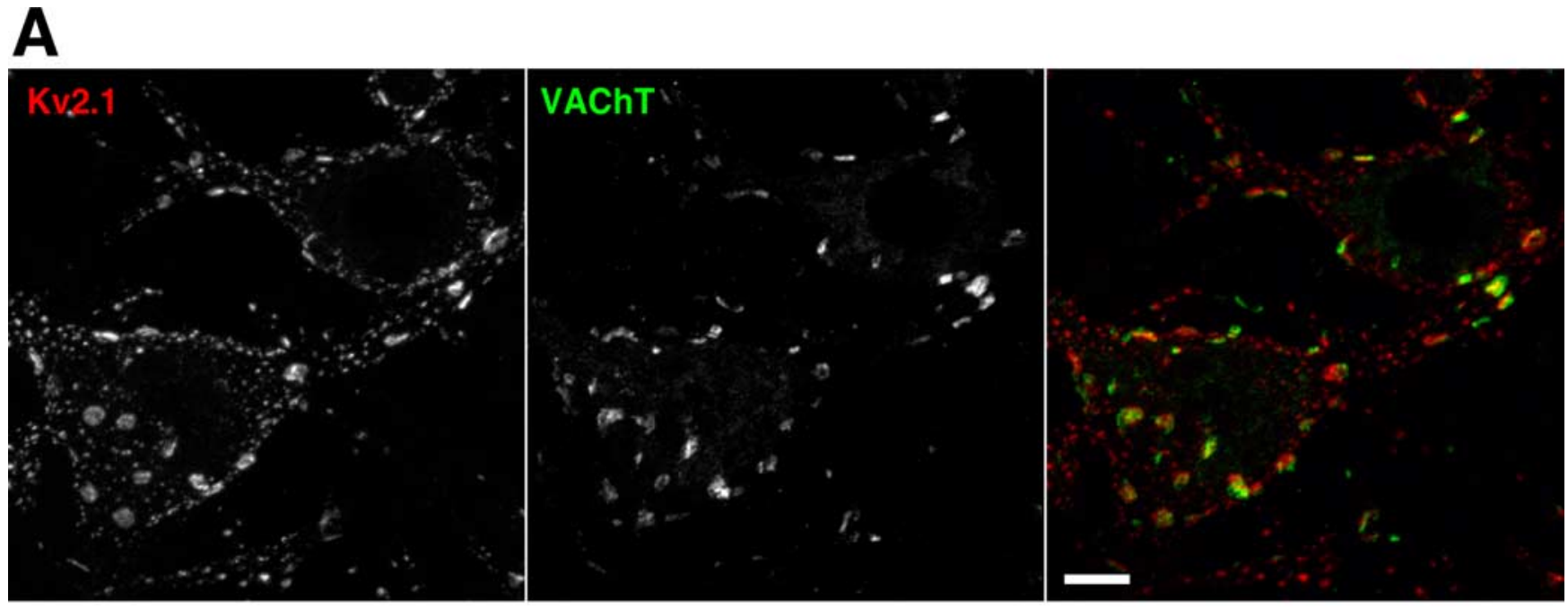

B
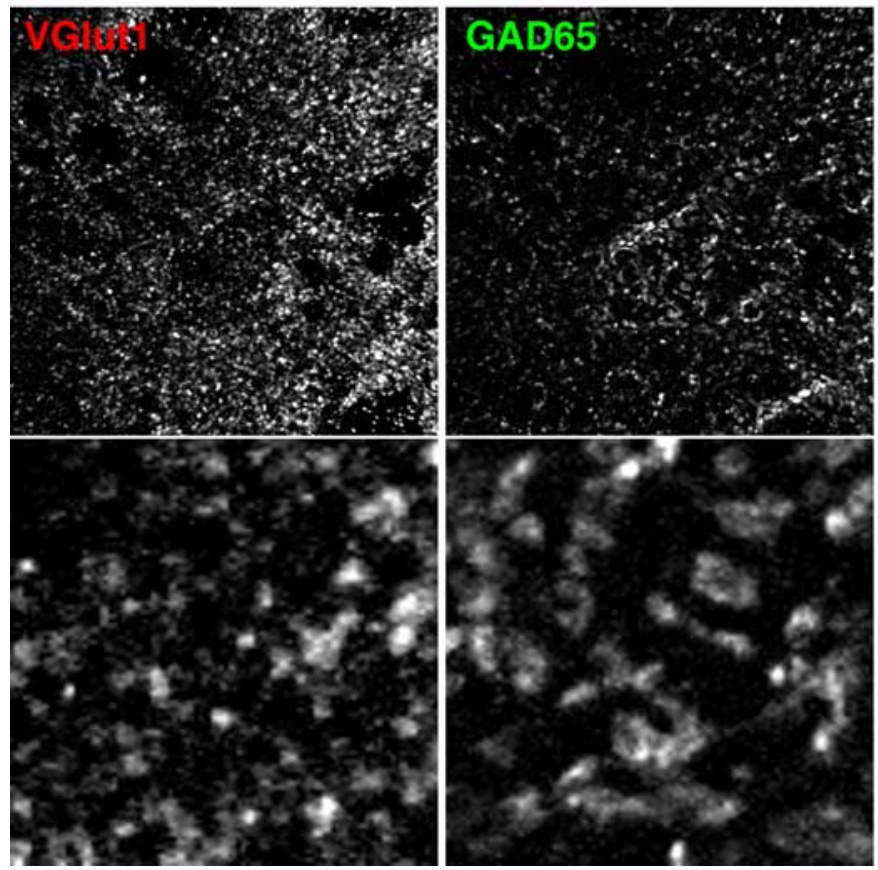
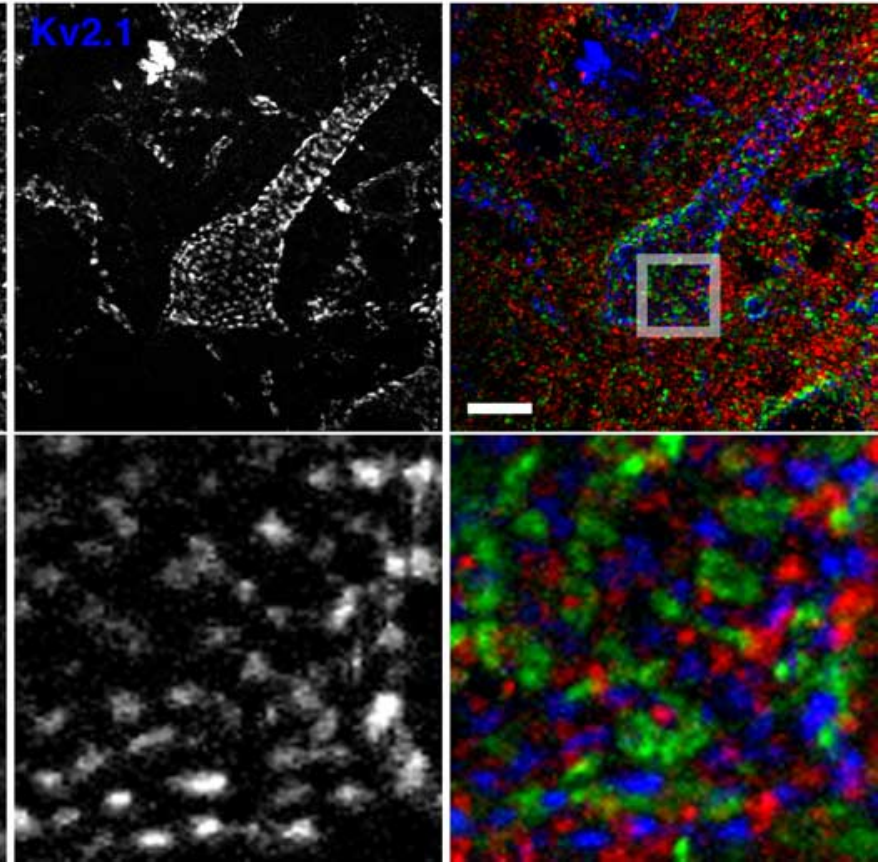

Figure 1. Extrasynaptic localization of Kv2.1 clusters in pyramidal neurons of rat cerebral cortex. A, Brain sections were stained for Kv2.1 (red) and VAChT (green). Motoneurons in the facial nucleus are shown. B, Brain sections were stained for Kv2.1 (blue) and presynaptic markers VGluT1 (red) and GAD65 (green). Pyramidal neurons in layer V of primary somatosensory cortex are shown. Bottom, Magnified images of the boxed area in the merged image. The overlay shows a clear segregation of Kv2.1 clusters from these synaptic markers. Images are composites from multiple optical sections. Scale bars, $10 \mu \mathrm{m}$.

detectable signals of GFAP and also an increase in the GFAP immunoreactivity in individual astrocytes (Fig. 4). These results suggest a dramatic change in the astrocytic function during acute brain ischemia.

To test the hypothesis that astrocytes are involved in ischemiainduced Kv2.1 modulation, we have established an in vitro model of "brain ischemia" using acute cortical slices in which the native brain structure is preserved, unlike in dissociated cultures. We found that Kv2.1 clusters in the extrasynaptic area seem to be fully established around P24 during development (supplemental Fig. 4, available at www.jneurosci.org as supplemental material). Thus, we chose to use young adult rats (P24-P30) for these experiments. Acute cortical slices ( $300 \mu \mathrm{m}$ thick) were incubated in ACSF containing $5 \mathrm{~mm}$ sodium azide and $5 \mathrm{~mm}$ 2-deoxy-D- glucose, which cause chemically induced energy depletion, CED (Misonou et al., 2005). Kv2.1 clustering and the phosphorylated state were well preserved in control acute slices in normal ACSF up to $1 \mathrm{~h}$ (data not shown). As shown in Figure 5, CED resulted in the time-dependent dephosphorylation of Kv2.1 (Fig. 5A) and dispersion of the channel clusters (Fig. $5 B$ ), similar to what we have previously observed in vivo and in cultured neurons (Misonou et al., 2005). Furthermore, the somatic delayed rectifier $\mathrm{K}^{+}$currents $\left(I_{\mathrm{K}}\right)$, which are largely composed of Kv2.1 channels (Guan et al., 2007), were also significantly altered during CED. Whole-cell patch-clamp recording from cortical pyramidal neurons in slices revealed that CED led to a large hyperpolarizing shift (control $G_{1 / 2}=+14.2 \pm 1.6 \mathrm{mV}$; CED $G_{1 / 2}=+0.2 \pm 2.9$ $\mathrm{mV} ; p=0.01$ in paired $t$ test; $n=4$ ) in the voltage dependence of 

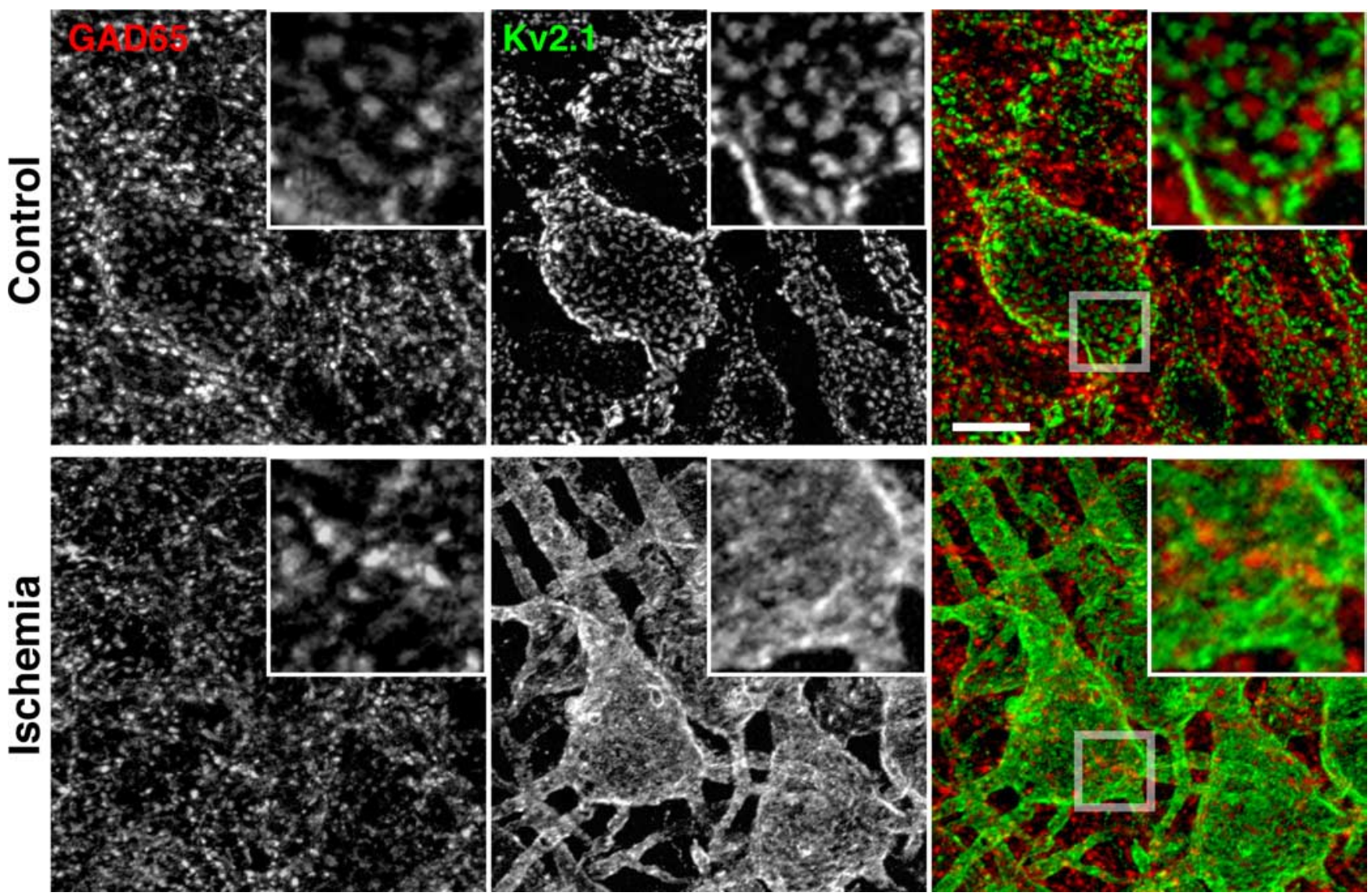

Figure 2. Translocation of Kv2.1 during acute brain ischemia. Brain sections from control and ischemic brains were stained for Kv2.1 (green) and GAD65 (red). The areas were chosen from the primary somatosensory cortex layer V. Insets, Magnified views of the boxed areas in the low-power images. Images are composites from multiple optical sections. Scale bar, $10 \mu \mathrm{m}$.

activation of $I_{\mathrm{K}}$, without any significant change in the slope $(k=$ 15.1 and 19.4, respectively; $p>0.05$ ) of the activation curve (Fig. $5 C$ ). As a result, the current amplitude of $I_{\mathrm{K}}$ at $-20 \mathrm{mV}$ depolarization was significantly increased from $219 \pm 46$ to $477 \pm 72 \mathrm{pA}$ $(p=0.045)$ after CED. These results are consistent with our previous findings in cultured neurons that dephosphorylation of Kv2.1 results in the potentiation of the delayed rectifier currents. Furthermore, we also confirmed that CED induced a substantial increase in the expression of GFAP (Fig. 5D) as observed similarly in vivo (Fig. 4).

To examine the effect of metabolic inhibition in astrocytes, we took advantage of fluorocitrate, which is widely used to inhibit the citric acid cycle selectively in astrocytes (Fonnum et al., 1997; Voloboueva et al., 2007). It has been shown that fluorocitrate causes the reduction of the intracellular ATP level in cultured astrocytes, reduces glutamate uptake, and eventually kills them (Voloboueva et al., 2007). We confirmed that fluorocitrate (50 $\mu \mathrm{M}, 18 \mathrm{~h}$ ) killed cultured cortical astrocytes as reported (data not shown). We focused on the analysis of the phosphorylation state of Kv2.1 because the level of phosphorylation is more quantifiable than the extent of channel clustering. The prediction from the hypothesis is that metabolic inhibition induced only in astrocytes by fluorocitrate is sufficient to induce Kv2.1 modulation. Indeed, we found that a 10 min treatment of slices with $50 \mu \mathrm{M}$ fluorocitrate alone effectively induced Kv2.1 dephosphorylation (the level of phosphorylated Kv2.1 at $\sim 125 \mathrm{kDa}$ reduced to $68 \pm$ $8.2 \%$ of control; $p<0.001 ; n=4)$ as well as CED $\operatorname{did}(66 \pm 6.6 \%$ of control; $p<0.001 ; n=4$ ) (Fig. $6 A, B$ ). These results suggest that ischemic stress in astrocyte is sufficient to rapidly cause Kv2.1 dephosphorylation in neurons.
Glutamate-dependent modulation of Kv2.1 during acute ischemic stress

It has been shown that in brain ischemia, there is an accumulation of glutamate in the extracellular space (Benveniste et al., 1984; Choi and Rothman, 1990). We have shown that glutamate stimulation induces Kv2.1 dephosphorylation by eliciting $\mathrm{Ca}^{2+}$ influx and the subsequent activation of calcineurin in cultured hippocampal neurons (Misonou et al., 2004). Together with the preliminary finding of another group that a nonselective inhibitor of glutamate transporter, TBOA (Shimamoto et al., 1998), induces Kv2.1 dephosphorylation in organotypic hippocampal slice culture (Mulholland et al., 2006), we hypothesized that the dysfunction of glutamate transporters in astrocytes during ischemia results in the accumulation of extracellular glutamate and glutamate-induced Kv2.1 modulation. The predictions are as follows: (1) glutamate transporter blockers mimic the ischemiainduced Kv2.1 modulation, and (2) the effect of CED is blocked by glutamate receptor antagonists. We confirmed that $100 \mu \mathrm{M}$ TBOA induced significant Kv2.1 dephosphorylation (the level of phosphorylated Kv2.1, $70 \pm 5.9 \%$ of control; $p<0.05 ; n=4$ ) (Fig. 6C). We then examined the effect of DHK, a specific inhibitor of GLT-1 (Arriza et al., 1994). As shown in Figure 6C, $100 \mu \mathrm{M}$ DHK for 10 min induced significant dephosphorylation of Kv2.1 $(67 \pm 4.9 \%$ of control; $p<0.05 ; n=4)$ in cortical slices. These results suggest that the pharmacological blockade of GLT-1 is sufficient to cause the Kv2.1 dephosphorylation.

To test whether there is an accumulation of glutamate during $\mathrm{CED}$, we examined the effect of glutamate receptor antagonists on the CED-induced Kv2.1 dephosphorylation. Slices were incubated with a mixture of antagonists (50 $\mu \mathrm{M}$ MK-801, $50 \mu \mathrm{M}$ AP-5, 


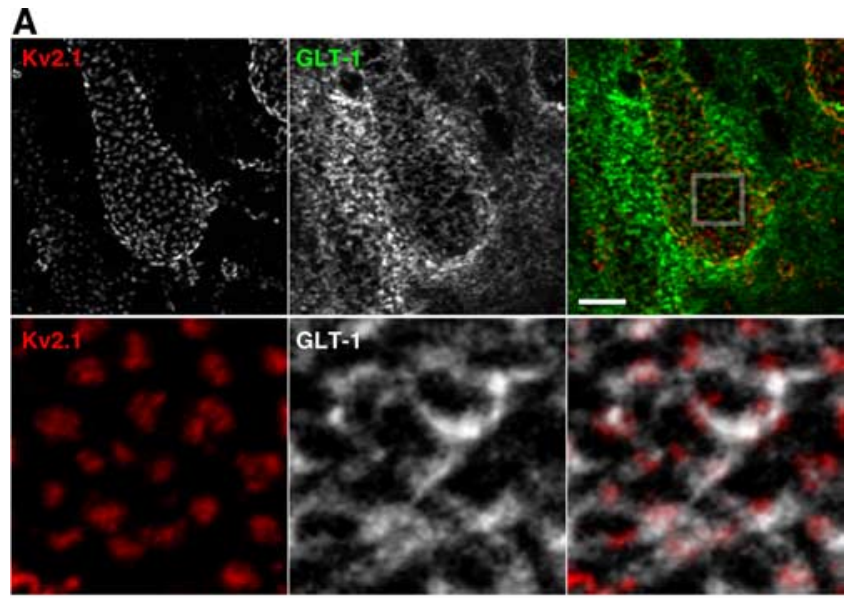

B
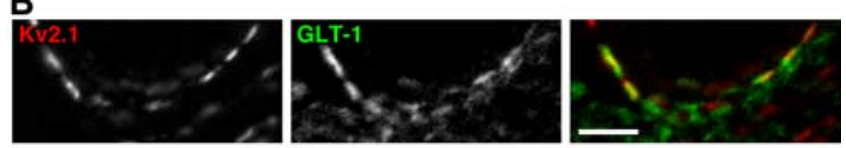

C
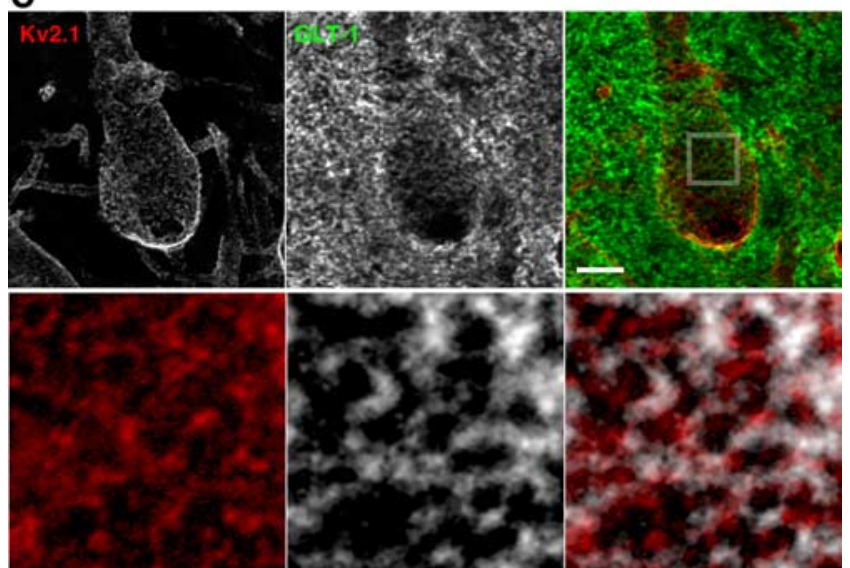

Figure 3. Association of Kv2.1 clusters and GLT-1 glial glutamate transporter. $\boldsymbol{A}$, Brain sections were stained for Kv2.1 (red) and glial glutamate transporter GLT-1 (green). Bottom, Magnified images of the boxed area in the merged image in the top row. GLT-1 staining is shown in white for better visualization. Images are composite from multiple optical sections. Scale bar, 10 $\mu \mathrm{m}$. B, High-magnification images of Kv2.1 and GLT-1 staining. Single optical sections clearly show the morphological association of these proteins at the edge of the neuron. Scale bar, 5 $\mu \mathrm{m}$. C, Kv2.1 dispersion relative to GLT-1-positive astrocytic processes. Brain sections from ischemic animals were stained for Kv2.1 (red) and GLT-1 (green). Bottom, Magnified images of the boxed area in the merged image in the top row. GLT-1 staining is shown in white. Scale bar, $10 \mu \mathrm{m}$

and $10 \mu \mathrm{M}$ CNQX) to block NMDA- and AMPA-type receptors for $10 \mathrm{~min}$ and then further subjected to CED in the presence of the antagonists. The antagonist treatment itself did not cause any detectable changes in the Kv2.1 phosphorylation state (data not shown). As shown in Figure $7 A$, the glutamate receptor antagonists completely blocked the Kv2.1 dephosphorylation induced by $10 \mathrm{~min}$ of CED. Importantly, the effects of the transporter blockers (TBOA and DHK) and fluorocitrate were also completely blocked by the antagonists (data not shown). Surprisingly, glutamate itself even at $100 \mu \mathrm{M}$ did not cause a significant change in Kv2.1 phosphorylation ( $95 \pm 4.8 \%$ of control) (Fig. $7 B$ ), although it is extremely effective to induce Kv2.1 dephosphorylation in culture (Misonou et al., 2004), where there are virtually no astrocytes (Misonou and Trimmer, 2005). Glutamate at $1 \mathrm{~mm}$ was able to induce substantial Kv2.1 dephosphorylation (Fig. $7 B)$.
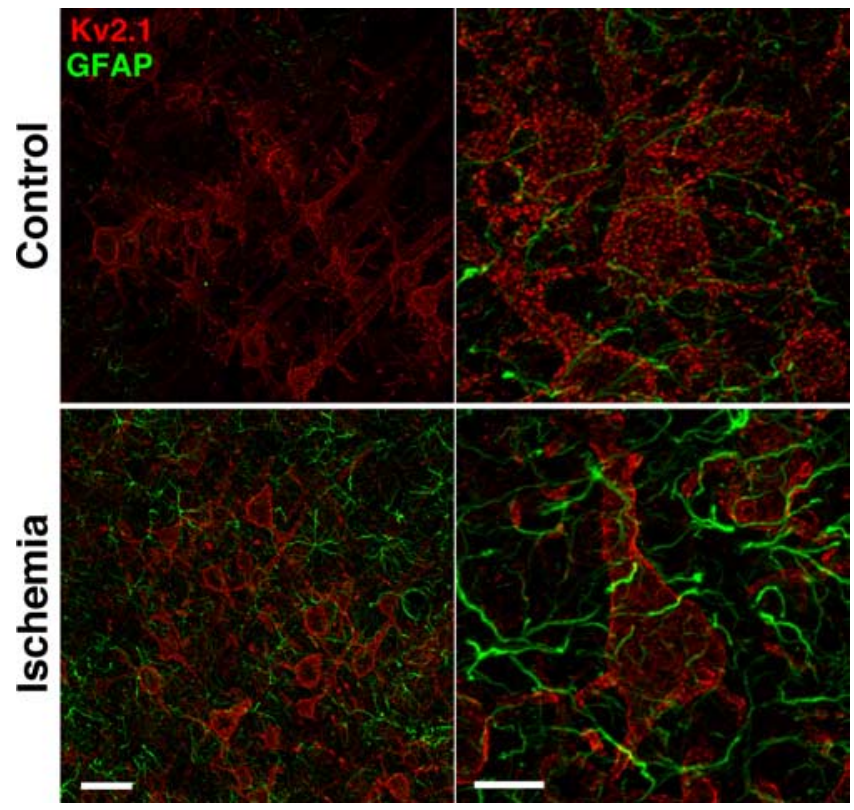

Figure 4. Morphological changes of astrocytes in the animal model of brain ischemia. Brain ischemia resulted not only in Kv2.1 cluster dispersion (red) but also in a marked increase in the level of GFAP immunoreactivity (green), indicating the activation of astrocytes. Right, Magnified images in layer $V$ of somatosensory cortex. Scale bars: left, $50 \mu \mathrm{m}$; right, $10 \mu \mathrm{m}$.

In contrast, NMDA, an agonist of the NMDA receptors, which is not transportable by glutamate transporters including GLT-1 (Garthwaite, 1985; Dowd et al., 1996; Herman and Jahr, 2007), at concentrations as low as $25 \mu \mathrm{M}$ induced significant dephosphorylation $(52 \pm 3.8 \%$ of control by $100 \mu \mathrm{M}$ NMDA; $p<0.001 ; n=$ $6)$ of Kv2.1 (Fig. 7B). These findings indicate not only that the glial glutamate transporter system is highly efficient in removing glutamate from the extracellular space in acute brain slices, as recently reported (Herman and Jahr, 2007), but also that an acute increase in glutamate release alone may not be sufficient to induce Kv2.1 modulation unless the glial glutamate uptake is impaired during ischemia.

The glutamate transporter system is coupled to the glutamine-glutamate cycle and thus to the citric acid cycle in astrocytes. It has been shown that the inhibition of glial glutamine synthetase by methionine sulfoxine (MSO) results in the accumulation of intracellular glutamate (Laake et al., 1995) and thus compromises the glutamate uptake in astrocytes (Jabaudon et al., 1999; Shaw et al., 1999; Cavelier and Attwell, 2005) in brain slice preparations. Therefore, we examined whether MSO induces Kv2.1 dephosphorylation in a glutamate-dependent manner. Slices were treated with $1.5 \mathrm{~mm}$ MSO for $30 \mathrm{~min}$ in the presence of $2 \mathrm{~mm}$ glutamine to keep the glutamate production in neurons. As shown in Figure 7C, MSO effectively induced Kv2.1 dephosphorylation. Importantly, the effects of MSO were also blocked by the glutamate receptor antagonists. These results support the hypothesis that brain ischemia results in the dysfunction of astrocytic glutamate transporters and extracellular glutamate accumulation, which ultimately leads to the functional modulation of Kv2.1 through glutamate receptor signaling.

\section{Discussion}

Ischemia-induced dephosphorylation of Kv2.1 in the neuroglial interaction

The major findings of this study are as follows: (1) Kv2.1 clusters are situated in the extrasynaptic area in rat cortical pyramidal 

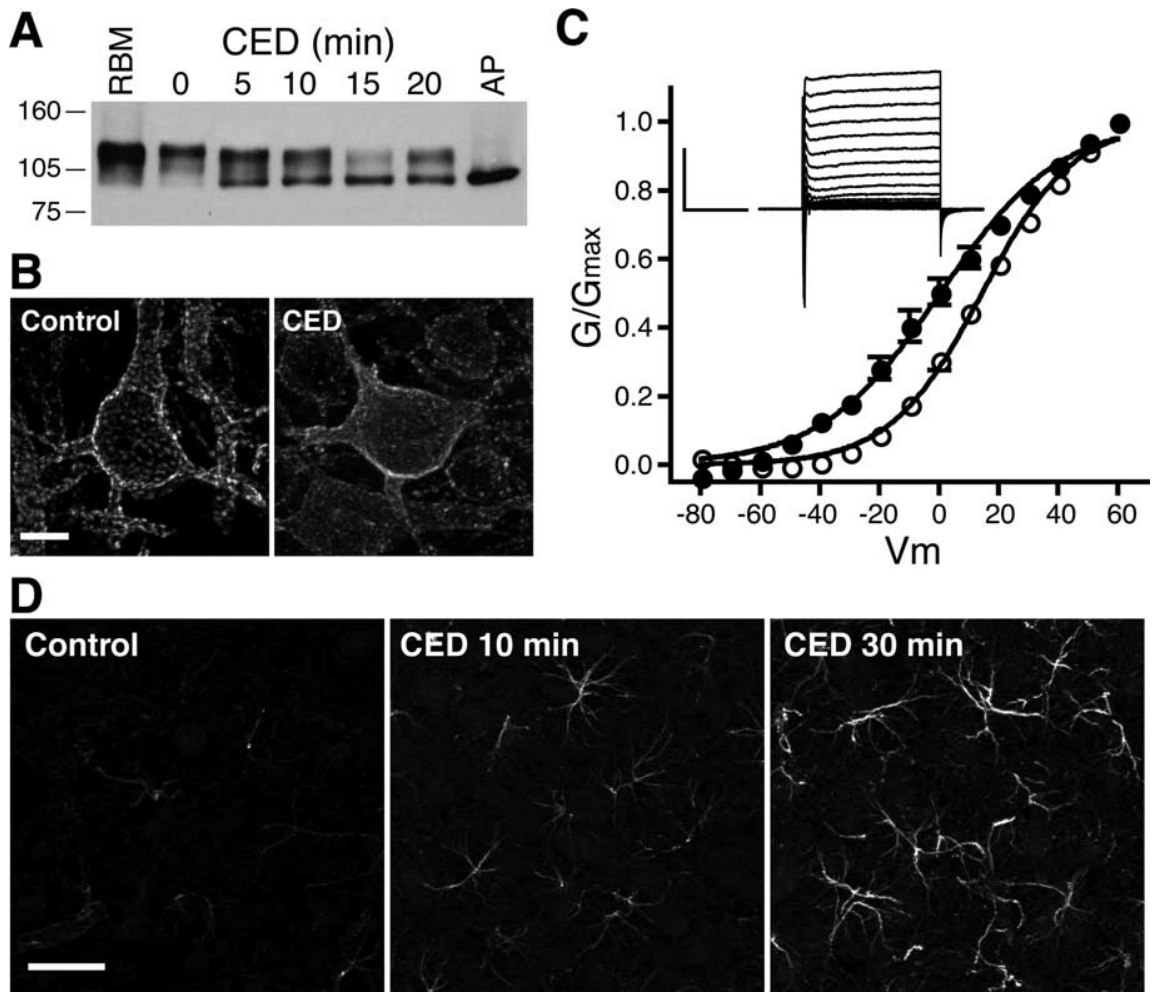

Figure 5. Kv2.1 dephosphorylation in the in vitro ischemia model. $\boldsymbol{A}, \mathrm{Kv} 2.1$ dephosphorylation induced by CED in acute cortical slices. Proteins were extracted from slices, separated by SDS-PAGE, and subjected to Western blotting. It should be noted that Kv2.1 appears as multiple bands because of multiple phosphorylated forms. RBM, Rat brain membrane fraction; AP, membrane fraction treated with alkaline phosphatase. AP treatment removes the higher-molecular-weight species and leaves only the fully dephosphorylated form of Kv2.1 ( 100 kDa). B, Dispersion of Kv2.1 clusters by CED ( $30 \mathrm{~min}$ ) in slices. It should be noted that $84 \pm$ $5 \%$ of control neurons exhibited clustered Kv2.1 and that only $13 \pm 5 \%$ of neurons had apparent clustered Kv2.1 in the CEDtreated slices $(n=3)$. Scale bar, $10 \mu \mathrm{m}$. C, CED alters the properties of neuronal $I_{K}$ current in cortical pyramidal neurons. Inset, Representative $I_{K}$ currents in a cortical pyramidal neuron recorded under whole-cell voltage clamp in an acute slice. Calibration: 2 $\mathrm{nA}, 100 \mathrm{~ms}$. The membrane potential was held at $-70 \mathrm{mV}$ and depolarized from the holding potential of $-70 \mathrm{mV}$ to voltages between -80 and $+60 \mathrm{mV}$ in $10 \mathrm{mV}$ increments for $200 \mathrm{~ms}$. A 30 ms prepulse to $-10 \mathrm{mV}$ was given before each test depolarization to eliminate transient $\mathrm{K}^{+}$current. The plot shows the conductance-voltage $(G-V)$ relationship of peak $I_{\mathrm{K}}$ currents recorded from neurons before (open circles) and after (filled circles) CED. Vm, Membrane potentials at $m V$. The current amplitude of $I_{\mathrm{K}}$ at $-20 \mathrm{mV}$ depolarization was also increased from $219 \pm 46$ to $477 \pm 72 \mathrm{pA}(p=0.045)$. $\boldsymbol{D}$, Morphological changes of astrocytes during CED. Sections from the treated slices were stained for GFAP. Scale bar, $50 \mu \mathrm{m}$.

neurons, (2) Kv2.1 clusters are adjacent to GLT-1-positive astrocytic processes, (3) brain ischemia rapidly causes changes in the cytoskeletal proteins in astrocytes, (4) metabolic inhibition in astrocytes is sufficient to cause Kv2.1 dephosphorylation in vitro, (5) the blockade of GLT-1 is sufficient to cause Kv2.1 dephosphorylation in vitro, (6) glutamate itself, even at $100 \mu \mathrm{M}$, is not able to induce Kv2.1 dephosphorylation in vitro, (7) nontransportable NMDA effectively causes Kv2.1 dephosphorylation in vitro, and (8) the effect of acute ischemic stress $(<10 \mathrm{~min})$ is mediated by glutamate and ionotropic glutamate receptors in vitro.

Based on these findings, we propose a model (Fig. 8). Ischemic stress rapidly affects astrocytes in the cerebral cortex and subsequently results in the dysfunction of glial glutamate transporters, particularly GLT-1, the predominant glutamate transporter in the cerebral cortex (Lehre et al., 1995; Regan et al., 2007). The compromised transporter system does not effectively clear up glutamate, which elicits a pathological accumulation of glutamate in the tight extracellular space. Glutamate activates ionotropic glutamate receptors and thus results in Kv2.1 dephosphorylation in neurons. The finding that the elevation of glutamate alone may not be sufficient to cause Kv2.1 dephosphorylation suggests that the dysfunction of glial glutamate transporters is necessary to efficiently induce the functional modulation of the Kv2.1 channels. Furthermore, ischemic stress eventually causes an intracellular $\mathrm{Ca}^{2+}$ release, presumably in neurons as we have previously observed in dissociated culture (Misonou et al., 2005). The released $\mathrm{Ca}^{2+}$ also contributes to the ischemia-induced Kv2.1 dephosphorylation, but only when the ischemic stress is prolonged. We and others have shown in cultured neurons that the bulk dephosphorylation of Kv2.1 during ischemia promotes the activation of the $I_{\mathrm{K}}$ channel. We have confirmed in the slice model that ischemia shifts Kv2.1 activation toward resting membrane potential, rendering it more easily activated, which then leads to dampening of the neuronal excitability. Therefore, this may represent a novel mechanism of the protective response of neurons to ischemic stress involving a voltage-gated potassium channel in the context of neuroglial interactions.

\section{Roles of glial glutamate transporters in} Kv2.1 modulation

Astrocytes express two major $\mathrm{Na}^{+} / \mathrm{K}^{+}$. dependent glutamate transporters, GLAST and GLT-1 (Palacín et al., 1998). These high-affinity glutamate transporters, particularly GLT-1, are important in removing spillover glutamate to achieve synapse-specific transmission (Huang and Bergles, 2004) in the cerebral cortex. Glutamate transporters are so efficient that they actively keep the ambient glutamate level in the extracellular space very low $(<100 \mathrm{~nm})$ (Herman and Jahr, 2007). This may not be the case in brain ischemia. There is compelling evidence that glutamate is accumulated in the extracellular space in an uncontrolled manner during ischemia (Benveniste et al., 1984; Choi and Rothman, 1990). Our results suggest that glutamate is accumulated to a level sufficient to cause Kv2.1 dephosphorylation during ischemia in vitro. We do not know where glutamate is derived from, synaptic and/or nonsynaptic origins. Growing evidence from specific pharmacology (Phillis et al., 2000) and from gene knock-out mice (Tanaka et al., 1997; Mitani and Tanaka, 2003) shows that the dysfunction of GLT-1 is largely responsible for the glutamate accumulation in acute ischemia (Hertz and Zielke, 2004). Our finding shows that inhibiting GLT-1 function with DHK and TBOA is sufficient to cause glutamate-dependent Kv2.1 dephosphorylation in vitro, although this does not exclude the possibility of glutamate release from astrocytes during ischemia (Tanaka et al., 1997; Phillis et al., 2000; Rossi et al., 2000; Mitani and Tanaka, 2003; Hertz and Zielke, 2004). It has been shown that the extracellular concentration of $\mathrm{K}^{+}$can be dramatically increased during brain ischemia (Astrup et al., 1977). Because GLT-1 uptakes glutamate in exchange for the efflux of $\mathrm{K}^{+}$, the increased extracellular $\mathrm{K}^{+}$may be able to cause the reversal of glutamate transport (Palacín et al., 1998; Rossi et al., 2007). The other glutamate transporters, in- 


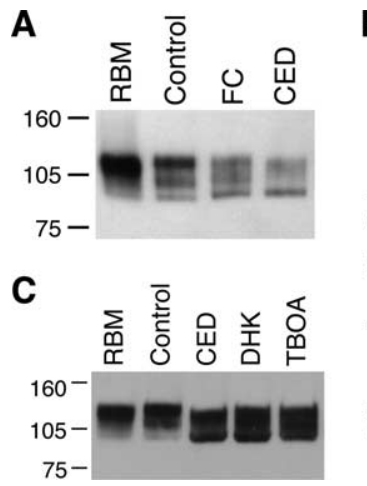

B

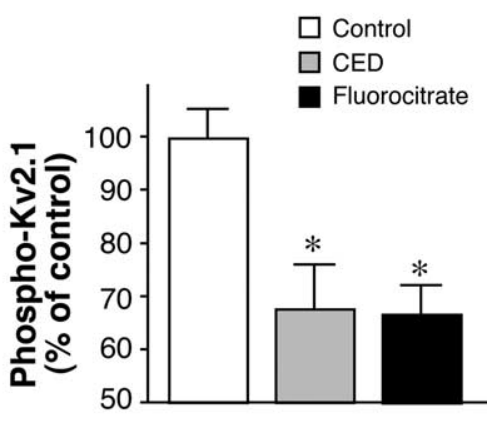

Figure 6. Kv2.1 dephosphorylation mediated by astrocytes. A, Kv2.1 dephosphorylation induced by fluorocitrate (FC). Slices were treated for 10 min with $50 \mu \mathrm{mFC}$ or with CED. RBM, Rat brain membrane fraction. $\boldsymbol{B}$, The levels of the highly phosphorylated form of Kv2.1 ( $\sim 125 \mathrm{kDa})$ were quantified and expressed as percentages of control. ${ }^{*} p<0.05$ compared with the control. C, Kv2.1 dephosphorylation induced by glutamate transporter blockers. Slices were treated for 10 min with $100 \mu \mathrm{m}$ DHK, $100 \mu \mathrm{M}$ TBOA, or CED.

cluding GLAST and a neuronal transporter EAAT3, may also be involved (Rossi et al., 2007). Astrocytes also express components required for exocytosis and can secrete glutamate to neurons (Bezzi et al., 2004), although the contribution of this mechanism in brain ischemia has not been fully addressed.

It has been unequivocally shown that the impairment of glutamate uptake is rapidly caused by energy depletion and results in the accumulation of glutamate within 1 min (Jabaudon et al., 2000). Multiple mechanisms have been implicated, including the aforementioned increase in the extracellular $\mathrm{K}^{+}$during ischemia (Leis et al., 2005) and the intracellular accumulation of $\mathrm{Na}^{+}$ (Allen et al., 2004). Both fluorocitrate and CED would result in the intracellular ATP depletion in astrocytes. This compromises many glial transporter systems, such as $\mathrm{Na}^{+} / \mathrm{K}^{+}$ATPase, and changes the intracellular ionic environment, such as $\mathrm{Na}^{+}$concentration, which is critical for the proper functioning of glutamate transporters (Allen et al., 2004; Rossi et al., 2007). Because the glutamine-glutamate cycle is also directly linked to the citric acid cycle (Nedergaard et al., 2002), the cessation of the cycle would also affect the glutamate uptake of astrocytes (Torp et al., 1991; Rossi et al., 2007), which is also implicated in our results that MSO induced the glutamate-dependent Kv2.1 dephosphorylation. However, the mechanism of transporter dysfunction requires further investigation.

\section{Possible mechanisms of Kv2.1 clustering in the extrasynaptic area}

Kv2.1 forms clusters in cultured neurons (Lim et al., 2000) or even in human embryonic kidney HEK293 cells (O'Connell and Tamkun, 2005; Mohapatra and Trimmer, 2006; O'Connell et al., 2006) without astrocytes and native synaptic structures. These findings indicate that Kv2.1 has an intrinsic property to form clusters in a proper cellular background (Kv2.1 is not clustered in COS- 1 cells). In fact, there is a signal sequence in the $\mathrm{C}$ terminus in which a mutation disrupts both the restricted localization and the cluster formation of Kv2.1 in cultured neurons (Lim et al., 2000). However, these two characteristics of Kv2.1 localization can be regulated independently because Kv2.1 dephosphorylation induced by glutamate stimulation and by ischemic stress causes dispersion of Kv2.1 clusters but retains the restricted localization (Misonou et al., 2004, 2005). Here we added another level of control in Kv2.1 localization. In the present study we show that Kv2.1 is clustered exclusively in the extrasynaptic area
A
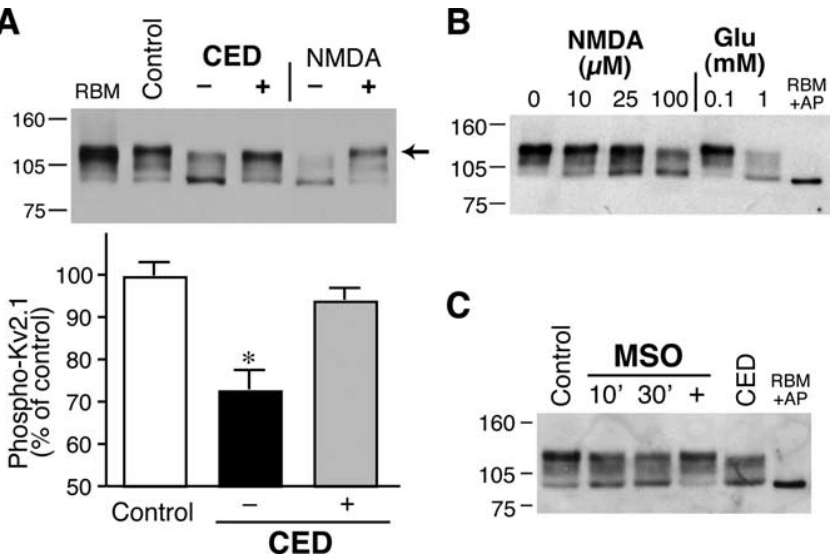

C

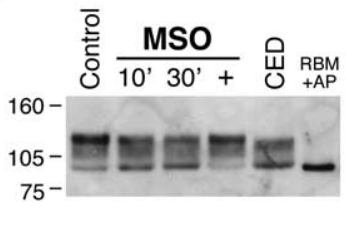

Figure 7. Glutamate-dependent modulation of Kv2.1 during ischemia. A, CED induced Kv2.1 dephosphorylation mediated by glutamate receptor signaling. Slices were pretreated without $(-)$ or with $(+)$ glutamate receptor antagonists for $10 \mathrm{~min}$ and then treated with CED or NMDA for $10 \mathrm{~min}$. The graph shows the quantified levels of the fully phosphorylated Kv2.1. ${ }^{*} p<0.05$ compared with the control. $\boldsymbol{B}$, NMDA, but not glutamate, induces rapid Kv2.1 dephosphorylation in vitro. Slices were treated with increasing concentrations of NMDA or with glutamate for $10 \mathrm{~min}$. C, Kv2.1 dephosphorylation induced by MSO. Slices were treated with $1.5 \mathrm{~mm}$ MSO or CED for 10-30 min. Some slices were pretreated with the glutamate receptor antagonists before MSO treatment (+). RBM, Rat brain membrane fraction; AP, membrane fraction treated with alkaline phosphatase.

and adjacent to GLT-1-positive astrocytic processes. This is in agreement with the previous immunoEM observation by Du et al. (1998) that many of the somatic Kv2.1 clusters are apposed to astrocytic membranes. Therefore, Kv2.1 localization is regulated at three levels: (1) restriction to the proximal part of a neuron, (2) clustering, and (3) positioning of clusters in the extrasynaptic area. Because the clustering can occur in the heterologous cells, it seems that the clustering and the extrasynaptic localization are separately regulated.

What is the mechanism of clustering? It has been convincingly shown that in HEK293 cells Kv2.1 clusters are formed and maintained by a molecular fence produced by actin cytoskeletons (O'Connell et al., 2006). The single particle tracking technique nicely shows that a Kv2.1 molecule in a cluster moves freely within the cluster but is bounced at the cluster perimeter. In these cells, Kv2.1 clusters are relatively dynamic, such that clusters are mobile and sometime fuse to each other. The situation might be different in neurons, particularly in the native brain structure. In our previous live-cell imaging with GFP-tagged Kv2.1 in cultured neurons, we found that Kv2.1 clusters in mature neurons are stable, such that clusters stay at the bottom of the cell where the cell adheres to an extracellular substrate (Misonou et al., 2004). In addition to this, the precise localization of Kv2.1 in the extrasynaptic area in neurons in vivo makes us speculate that there are proteins that cluster and anchor Kv2.1 to those specific areas of neuronal plasma membrane. The contrasting synaptic localization of Kv2.1 in motoneurons (Muennich and Fyffe, 2004) also supports the idea that the position of Kv2.1 clusters can vary in different types of cells, depending on the anchor proteins expressed. However, the actin fence mechanism may still be involved, because the clustering and the extrasynaptic localization can be separated in the early development (see supplemental Fig. 3 , available at www.jneurosci.org as supplemental material). Identification of the proteins that interact with Kv2.1 in the brain would help our understanding of the mechanism of the unique localization of this channel.

Why does Kv2.1 have to be clustered? In the electrophysiolog- 


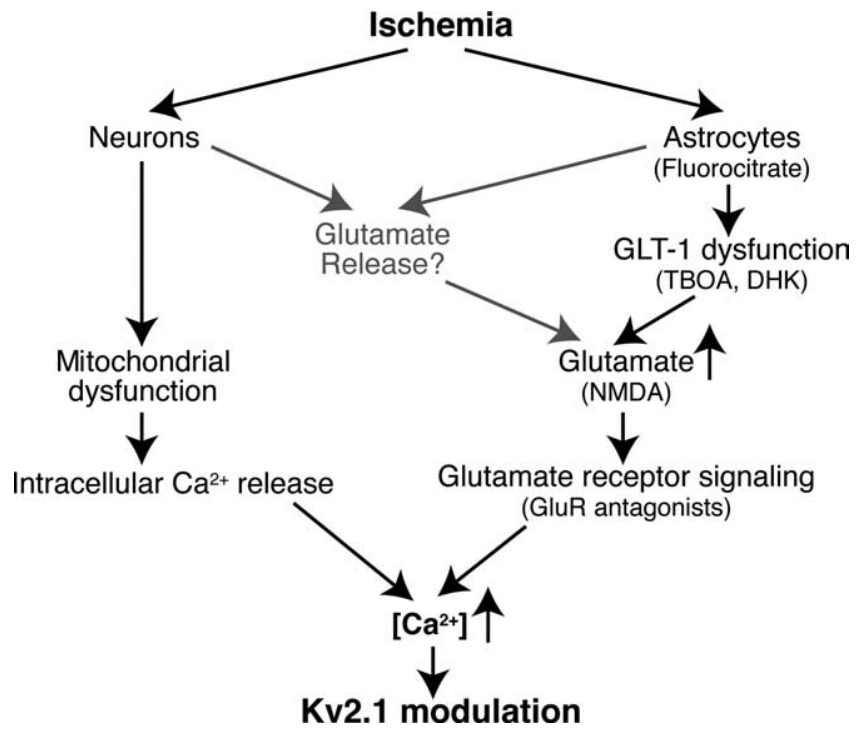

Figure 8. Schematic diagram of our model. During brain ischemia, the signaling pathway mediated by astrocytes (right) takes place immediately $(<5 \mathrm{~min})$, and then later, the left pathway will also be driven to cause Kv2.1 modulation. They both ultimately induce the increase in the intracellular $\mathrm{Ca}^{2+}$ level, followed by Kv2.1 modulation (dephosphorylation, dispersion, and activation). The text in the parentheses indicates the experimental supports of the signaling pathways. See Discussion for more detail.

ical sense, the channels do not have to be clustered to affect the membrane potential of presumably isoelectric somatic membrane. Kv2.1 may be clustered for some other reasons. We speculate that Kv2 channel clusters are strategically situated at the junction between the astrocytic and neuronal membranes to achieve rapid dephosphorylation and the activation of the channel function during brain ischemia by sensing the dysfunction of astrocytes through glutamate, with a concerted action of neuronal glutamate signaling. Dispersion of Kv2.1 clusters after calcineurin-mediated dephosphorylation could provide a mechanism for more efficient reversibility and recovery, by removing Kv2.1 from this region of local $\mathrm{Ca}^{2+}$ signaling.

\section{References}

Allen NJ, Káradóttir R, Attwell D (2004) Reversal or reduction of glutamate and GABA transport in CNS pathology and therapy. Pflugers Arch 449:132-142.

Arriza JL, Fairman WA, Wadiche JI, Murdoch GH, Kavanaugh MP, Amara SG (1994) Functional comparisons of three glutamate transporter subtypes cloned from human motor cortex. J Neurosci 14:5559-5569.

Astrup J, Symon L, Branston NM, Lassen NA (1977) Cortical evoked potential and extracellular $\mathrm{K}+$ and $\mathrm{H}+$ at critical levels of brain ischemia. Stroke 8:51-57.

Ballanyi K (2004) Protective role of neuronal KATP channels in brain hypoxia. J Exp Biol 207:3201-3212.

Benveniste H, Drejer J, Schousboe A, Diemer NH (1984) Elevation of the extracellular concentrations of glutamate and aspartate in rat hippocampus during transient cerebral ischemia monitored by intracerebral microdialysis. J Neurochem 43:1369-1374.

Bezzi P, Gundersen V, Galbete JL, Seifert G, Steinhäuser C, Pilati E, Volterra A (2004) Astrocytes contain a vesicular compartment that is competent for regulated exocytosis of glutamate. Nat Neurosci 7:613-620.

Cai X, Liang CW, Muralidharan S, Kao JP, Tang CM, Thompson SM (2004) Unique roles of SK and Kv4.2 potassium channels in dendritic integration. Neuron 44:351-364.

Cavelier P, Attwell D (2005) Tonic release of glutamate by a DIDS-sensitive mechanism in rat hippocampal slices. J Physiol 564:397-410.

Choi DW, Rothman SM (1990) The role of glutamate neurotoxicity in hypoxic-ischemic neuronal death. Annu Rev Neurosci 13:171-182.

Danbolt NC (2001) Glutamate uptake. Prog Neurobiol 65:1-105.
Dowd LA, Coyle AJ, Rothstein JD, Pritchett DB, Robinson MB (1996) Comparison of $\mathrm{Na}+$-dependent glutamate transport activity in synaptosomes, C6 glioma, and Xenopus oocytes expressing excitatory amino acid carrier 1 (EAAC1). Mol Pharmacol 49:465-473.

Du J, Tao-Cheng JH, Zerfas P, McBain CJ (1998) The K+ channel, Kv2.1, is apposed to astrocytic processes and is associated with inhibitory postsynaptic membranes in hippocampal and cortical principal neurons and inhibitory interneurons. Neuroscience 84:37-48.

Fonnum F, Johnsen A, Hassel B (1997) Use of fluorocitrate and fluoroacetate in the study of brain metabolism. Glia 21:106-113.

Garthwaite J (1985) Cellular uptake disguises action of L-glutamate on $\mathrm{N}$-methyl-D-aspartate receptors. With an appendix: diffusion of transported amino acids into brain slices. Br J Pharmacol 85:297-307.

Guan D, Tkatch T, Surmeier DJ, Armstrong WE, Foehring RC (2007) Kv2 subunits underlie slowly inactivating potassium current in neocortical pyramidal neurons. J Physiol 581:941-960.

Herman MA, Jahr CE (2007) Extracellular glutamate concentration in hippocampal slice. J Neurosci 27:9736-9741.

Hertz L, Zielke HR (2004) Astrocytic control of glutamatergic activity: astrocytes as stars of the show. Trends Neurosci 27:735-743.

Huang YH, Bergles DE (2004) Glutamate transporters bring competition to the synapse. Curr Opin Neurobiol 14:346-352.

Jabaudon D, Shimamoto K, Yasuda-Kamatani Y, Scanziani M, Gähwiler BH, Gerber U (1999) Inhibition of uptake unmasks rapid extracellular turnover of glutamate of nonvesicular origin. Proc Natl Acad Sci U S A 96:8733-8738.

Jabaudon D, Scanziani M, Gähwiler BH, Gerber U (2000) Acute decrease in net glutamate uptake during energy deprivation. Proc Natl Acad Sci U S A 97:5610-5615.

Kash SF, Johnson RS, Tecott LH, Noebels JL, Mayfield RD, Hanahan D, Baekkeskov S (1997) Epilepsy in mice deficient in the 65-kDa isoform of glutamic acid decarboxylase. Proc Natl Acad Sci U S A 94:14060-14065.

Laake JH, Slyngstad TA, Haug FM, Ottersen OP (1995) Glutamine from glial cells is essential for the maintenance of the nerve terminal pool of glutamate: immunogold evidence from hippocampal slice cultures. J Neurochem 65:871-881.

Lehre KP, Levy LM, Ottersen OP, Storm-Mathisen J, Danbolt NC (1995) Differential expression of two glial glutamate transporters in the rat brain: quantitative and immunocytochemical observations. J Neurosci 15:1835-1853.

Leis JA, Bekar LK, Walz W (2005) Potassium homeostasis in the ischemic brain. Glia 50:407-416.

Lim ST, Antonucci DE, Scannevin RH, Trimmer JS (2000) A novel targeting signal for proximal clustering of the $\mathrm{Kv} 2.1 \mathrm{~K}+$ channel in hippocampal neurons. Neuron 25:385-397.

Misonou H, Trimmer JS (2005) A primary culture system for biochemical analyses of neuronal proteins. J Neurosci Methods 144:165-173.

Misonou H, Mohapatra DP, Park EW, Leung V, Zhen D, Misonou K, Anderson AE, Trimmer JS (2004) Regulation of ion channel localization and phosphorylation by neuronal activity. Nat Neurosci 7:711-718.

Misonou H, Mohapatra DP, Menegola M, Trimmer JS (2005) Calcium- and metabolic state-dependent modulation of the voltage-dependent Kv2.1 channel regulates neuronal excitability in response to ischemia. J Neurosci 25:11184-11193.

Misonou H, Menegola M, Mohapatra DP, Guy LK, Park KS, Trimmer JS (2006) Bidirectional activity-dependent regulation of neuronal ion channel phosphorylation. J Neurosci 26:13505-13514.

Mitani A, Tanaka K (2003) Functional changes of glial glutamate transporter GLT-1 during ischemia: an in vivo study in the hippocampal CA1 of normal mice and mutant mice lacking GLT-1. J Neurosci 23:7176-7182.

Mohapatra DP, Trimmer JS (2006) The Kv2.1 C terminus can autonomously transfer Kv2.1-like phosphorylation-dependent localization, voltage-dependent gating, and muscarinic modulation to diverse $\mathrm{Kv}$ channels. J Neurosci 26:685-695.

Muennich EA, Fyffe RE (2004) Focal aggregation of voltage-gated, Kv2.1 subunit-containing, potassium channels at synaptic sites in rat spinal motoneurones. J Physiol 554:673-685.

Mulholland P, Carpenter-Hyland E, Chandler L (2006) Extrasynaptic NMDA receptors couple to dephosphorylation of voltage-dependent Kv2.1 potassium channels. Soc Neurosci Abstr 32:530.11.

Murakoshi H, Trimmer JS (1999) Identification of the Kv2.1 $\mathrm{K}^{+}$channel as 
a major component of the delayed rectifier $\mathrm{K}^{+}$current in rat hippocampal neurons. J Neurosci 19:1728-1735.

Nedergaard M, Takano T, Hansen AJ (2002) Beyond the role of glutamate as a neurotransmitter. Nat Rev Neurosci 3:748-755.

O'Connell KM, Tamkun MM (2005) Targeting of voltage-gated potassium channel isoforms to distinct cell surface microdomains. J Cell Sci 118:2155-2166.

O'Connell KM, Rolig AS, Whitesell JD, Tamkun MM (2006) Kv2.1 potassium channels are retained within dynamic cell surface microdomains that are defined by a perimeter fence. J Neurosci 26:9609-9618.

Pal S, Hartnett KA, Nerbonne JM, Levitan ES, Aizenman E (2003) Mediation of neuronal apoptosis by Kv2.1-encoded potassium channels. J Neurosci 23:4798-4802.

Palacín M, Estévez R, Bertran J, Zorzano A (1998) Molecular biology of mammalian plasma membrane amino acid transporters. Physiol Rev 78:969-1054.

Phillis JW, Ren J, O’Regan MH (2000) Transporter reversal as a mechanism of glutamate release from the ischemic rat cerebral cortex: studies with DL-threo-beta-benzyloxyaspartate. Brain Res 880:224.

Regan MR, Huang YH, Kim YS, Dykes-Hoberg MI, Jin L, Watkins AM, Bergles DE, Rothstein JD (2007) Variations in promoter activity reveal a differential expression and physiology of glutamate transporters by glia in the developing and mature CNS. J Neurosci 27:6607-6619.

Rhodes KJ, Trimmer JS (2006) Antibodies as valuable neuroscience research tools versus reagents of mass distraction. J Neurosci 26:8017-8020.

Rossi DJ, Oshima T, Attwell D (2000) Glutamate release in severe brain ischaemia is mainly by reversed uptake. Nature 403:316-321.

Rossi DJ, Brady JD, Mohr C (2007) Astrocyte metabolism and signaling during brain ischemia. Nat Neurosci 10:1377-1386.

Scannevin RH, Murakoshi H, Rhodes KJ, Trimmer JS (1996) Identification of a cytoplasmic domain important in the polarized expression and clustering of the Kv2.1 K+ channel. J Cell Biol 135:1619-1632.

Shaw CA, Bains JS, Pasqualotto BA, Curry K (1999) Methionine sulfoximine shows excitotoxic actions in rat cortical slices. Can J Physiol Pharmacol 77:871-877.

Shimamoto K, Lebrun B, Yasuda-Kamatani Y, Sakaitani M, Shigeri Y, Yu- moto N, Nakajima T (1998) DL-threo-beta-benzyloxyaspartate, a potent blocker of excitatory amino acid transporters. Mol Pharmacol 53:195-201.

Sullivan R, Rauen T, Fischer F, Wiessner M, Grewer C, Bicho A, Pow DV (2004) Cloning, transport properties, and differential localization of two splice variants of GLT-1 in the rat CNS: implications for CNS glutamate homeostasis. Glia 45:155-169.

Takuma K, Baba A, Matsuda T (2004) Astrocyte apoptosis: implications for neuroprotection. Prog Neurobiol 72:111-127.

Tanaka E, Yamamoto S, Kudo Y, Mihara S, Higashi H (1997) Mechanisms underlying the rapid depolarization produced by deprivation of oxygen and glucose in rat hippocampal CA1 neurons in vitro. J Neurophysiol 78:891-902.

Tokumine J, Kakinohana O, Cizkova D, Smith DW, Marsala M (2003) Changes in spinal GDNF, BDNF, and NT-3 expression after transient spinal cord ischemia in the rat. J Neurosci Res 74:552-561.

Torp R, Andiné P, Hagberg H, Karagülle T, Blackstad TW, Ottersen OP (1991) Cellular and subcellular redistribution of glutamate-, glutamineand taurine-like immunoreactivities during forebrain ischemia: a semiquantitative electron microscopic study in rat hippocampus. Neuroscience 41:433-447.

Trimmer JS (1991) Immunological identification and characterization of a delayed rectifier $\mathrm{K}+$ channel polypeptide in rat brain. Proc Natl Acad Sci U S A 88:10764-10768.

Voloboueva LA, Suh SW, Swanson RA, Giffard RG (2007) Inhibition of mitochondrial function in astrocytes: implications for neuroprotection. J Neurochem 102:1383-1394.

Wei L, Yu SP, Gottron F, Snider BJ, Zipfel GJ, Choi DW (2003) Potassium channel blockers attenuate hypoxia- and ischemia-induced neuronal death in vitro and in vivo. Stroke 34:1281-1286.

Yamada K, Ji JJ, Yuan H, Miki T, Sato S, Horimoto N, Shimizu T, Seino S, Inagaki N (2001) Protective role of ATP-sensitive potassium channels in hypoxia-induced generalized seizure. Science 292:1543-1546.

Yuan LL, Adams JP, Swank M, Sweatt JD, Johnston D (2002) Protein kinase modulation of dendritic $\mathrm{K}^{+}$channels in hippocampus involves a mitogen-activated protein kinase pathway. J Neurosci 22:4860-4868. 